\title{
DESIGN OF THE INJECTION KICKER MAGNET SYSTEM FOR CERN'S 14 TeV PROTON COLLIDER LHC
}

\author{
L. Ducimetière, U. Jansson, G.H. Schröder, E.B. Vossenberg \\ CERN SL Division, CH-1211 Geneva 23, Switzerland \\ M.J. Barnes, G.D. Wait \\ TRIUMF, 4004 Wesbrook Mall, Vancouver, B.C., Canada V6T 2A3
}

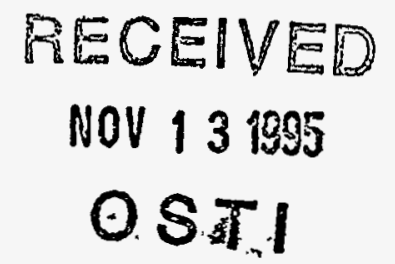

\begin{abstract}
Two counter-rotating proton beams will be injected into the LHC at an energy of $450 \mathrm{GeV}$ by two kicker magnet systems, producing magnetic field pulses of approximately 750 ns rise time and $6.6 \mu$ slat top duration. To avoid dilution of the beam emittance during injection, a stringent design requirement of the system is a flat top ripple of the magnetic field of less than $\pm 0.5 \%$. Both injection systems are composed of 4 travelling wave kicker magnets of $2.17 \mathrm{~m}$ length each, powered by pulse forming networks (PFN's) and matched to their characteristic impedance. To achieve the high required kick strength of $1.2 \mathrm{Tm}$, for a compact and cost efficient design, a comparably low characteristic impedance of $5 \Omega$ has been chosen. The electrical circuit of the system is being designed with the help of PSpice computer modelling. Most known parasitic elements are included in the model to obtain a realistic pulse response prediction. The present paper reports on design and modeling results of the LHC injection kicker magnet system that has several novel and demanding design requirements.
\end{abstract}

\section{Introduction}

In December 94 CERN, the European Laboratory for Particle Physics, was authorised to start construction of a new accelerator, the Large Hadron Collider (LHC). This machine will bring protons into head-on collisions at an energy of $14 \mathrm{TeV}$, higher than ever achieved before. It will also provide lead ion collisions at an energy of $1148 \mathrm{TeV}$ and could later be transformed into an electron-proton collider.

The LHC will make maximum use of CERN's existing installations. It will be located in the $27 \mathrm{~km}$ long tunnel of LEP, the Large Electron Positron Collider, and filled from a pre-accelerator, the $450 \mathrm{GeV}$ Super Proton Synchrotron (SPS) which itself is the last element in a chain of injector machines. Superconducting magnets of a novel, compact design will be employed, with 2 horizontally separated channels for the 2 counter-rotating beams which can collide in up to 4 interaction points. Each beam will have a circulating current of more than $0.5 \mathrm{~A}$, distributed in 2835 bunches of $10^{11}$ particles at $25 \mathrm{~ns}$ spacing. The beam channels will be filled by 12 batches of protons at $450 \mathrm{GeV}$, injected successively along the machine circumference.

\section{System requirements and parameters}

Injection is carried out in the horizontal plane by means of a copper septum magnet followed by a fast pulsed kicker system. The injected beam approaches the kicker at an angle of $0.8 \mathrm{mrad}$, requiring a total kick strength of $1.2 \mathrm{Tm}$ for deflection onto the central machine orbit. The collider will be filled with 11 batches of $6.5 \mu \mathrm{s}$ duration and 1 batch of $4.25 \mu \mathrm{s}$ duration, each to be deposited in one twelfth of the machine circumference, $7.14 \mu \mathrm{s}$. This leaves 11 gaps of $0.91 \mu \mathrm{s}$ for the kick rise time and one gap of $3.16 \mu \mathrm{s}$ for the kick fall time. The latter gap will also be used for the rise time of the beam dumping system.

The reflections and the flat top ripple of the field pulse must be lower than $\pm 0.5 \%$. This very demanding requirement is needed to limit the beam emittance blow-up due to injection oscillations. The pulse repetition time is $15 \mathrm{~s}$, imposed by the duration of the SPS acceleration cycle.

Making allowance for timing imprecision and jitter, the inflector system will be designed with a $0.5 \%$ to $99.5 \%$ rise time of $0.75 \mu \mathrm{s}$, an adjustable flat top of maximum duration of $6.6 \mu \mathrm{s}$ and a $99.5 \%$ to $0.5 \%$ fall time of $3.0 \mu \mathrm{s}$.

\section{Design strategy}

A carefully matched high bandwidth system is needed to obtain the stringent pulse response requirements. The system is therefore composed of a multi-cell PFN and a multi-cell travelling wave kicker magnet, connected by a 
matched transmission line and terminated by a matched resistor. To obtain a compact and cost efficient design a rather low system impedance (Z) of $5 \Omega$ has been chosen. The design voltage is $60 \mathrm{kV}$, as in most SPS kicker installations, allowing the use of several proven components such as thyratron housings, transmission line connectors and termination resistors. Fig. 1 gives the basic circuit diagram.

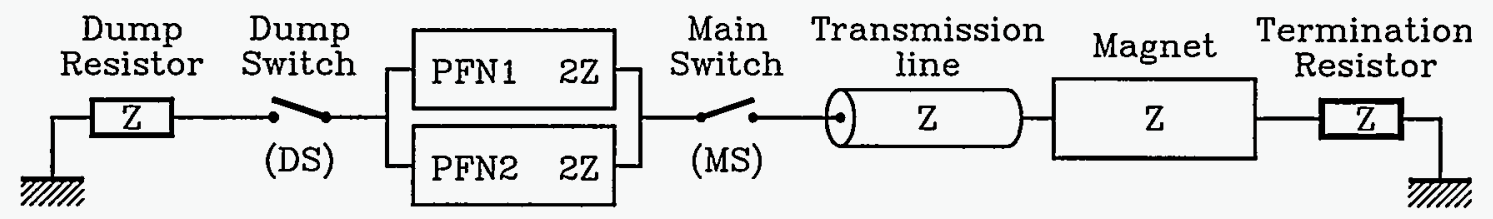

Fig. 1: Basic circuit diagram

Previous experience has shown that adjusting individual inductances of a PFN does not allow one to achieve a flat top ripple smaller than about $\pm 1 \%{ }^{[1]}$. For this reason the inductances will be wound as a continuous straight and rigid coil with constant and high precision pitch to guarantee the same self inductance and mutual coupling coefficient for all cells of the PFN. A characteristic impedance of $5 \Omega$ with adequate frequency response requires cell inductances of less than $1 \mu \mathrm{H}$. Such small inductances cannot be built cost efficiently with the required high precision ${ }^{[2]}$. It has therefore been decided to employ two PFN's of $10 \Omega$ impedance to be connected in parallel, with the following advantages:

- more acceptable dimension of the inductances ( $154 \mathrm{~mm}$ long, $\varnothing 80 \mathrm{~mm}, 7$ turns per cell);

- better mutual coupling between adjacent cells (smoothing effect);

- shorter overall length of the PFN due to smaller capacitors.

Table I summarizes the main parameters of each injection system. It is intended to employ three-gap CX 1171 thyratrons from EEV ${ }^{[3]}$ as power switches. In order to vary the pulse duration, a main switch (MS) and a dump switch (DS) are required, one at each end of the PFN. The ferrite loaded kicker magnet will have a C-configuration to allow earthing of the coaxial cable input connection and the output connection to the termination resistor. To achieve a low beam impedance it is intended to place a thin ceramic tube equipped with copper stripes and decoupling capacitors into the magnet aperture.

\begin{tabular}{|l|rl|}
\hline Number of magnets per system & 4 \\
\hline Kick strength per magnet & $0.3 \quad \mathrm{~T} . \mathrm{m}$ \\
\hline Characteristic impedance & 5 & $\Omega$ \\
\hline Charging voltage & $60 \mathrm{kV}$ \\
\hline Pulse current & 6 & $\mathrm{kA}$ \\
\hline Kick flat top ripple & $< \pm 0.5 \mathrm{\%}$ \\
\hline Kick flat top duration & $4.25 \& 6.6 \mu \mathrm{s}$ \\
\hline Kick rise time 0.5\%-99.5\% & $0.75 \mu \mathrm{s}$ \\
\hline Kick fall time 99.5\%-0.5\% & $3.0 \mathrm{\mu s}$ \\
\hline Repetition time & $15 \mathrm{~s}$ \\
\hline
\end{tabular}

Table I: System requirements and parameters

\section{Modelling and simulation results}

\section{General}

The electrical circuit was simulated using the PSpice ${ }^{[4]}$ analysis package. In order to obtain a realistic transient response most known parasitics were modelled. The rise and fall time definitions utilised for the theoretical studies are $0.1 \%$ to $99.9 \%$, with a reduced ripple tolerance of $\pm 0.1 \%$ to make allowance for mechanical tolerances during construction, fast changes of components due to pulsing and slow changes due to ageing. The process of determining component values, which result in an acceptable field rise-time, fall-time, and ripple, has been carried out in several stages. The optimisation process was done both by making educated guesses at component values, and using the PSpice optimizer ${ }^{[4]}$.

Initially the rising-edge and flat-top of the field were studied and brought within specification by optimising component values within the PFN: for this process the termination resistors and the kicker magnet have been treated as ideal components, and the CX 1171 thyratrons were simulated using a single-gap model ${ }^{[5]}$. The second stage of the process was to optimise component values, and add impedance matching networks, at the DS end of the PFN, to bring the fall-time and post-pulse period within specification. Subsequently the optimised PFN and impedance matching networks were simulated and the idealised representation of the kicker magnet was replaced with a realistic mathematical model of the travelling-wave magnet, which also included stripes and decoupling capacitors of the ceramic beam-pipe. 


\section{DISCLAIMER}

This report was prepared as an account of work sponsored by an agency of the United States Government. Neither the United States Government nor any agency thereof, nor any of their employees, make any warranty, express or implied, or assumes any legal liability or responsibility for the accuracy, completeness, or usefulness of any information, apparatus, product, or process disclosed, or represents that its use would not infringe privately owned rights. Reference herein to any specific commercial product, process, or service by trade name, trademark, manufacturer, or otherwise does not necessarily constitute or imply its endorsement, recommendation, or favoring by the United States Government or any agency thereof. The views and opinions of authors expressed herein do not necessarily state or reflect those of the United States Government or any agency thereof. 


\section{DISCLAIMER}

Portions of this document may be illegible in electronic image products. Images are produced from the best available original document. 


\section{Pulse Forming Network}

- Central cells

Preliminary calculations showed that about 25 cells were required for both the PFN's and the magnet, to obtain the required pulse characteristics. For practical reasons (availability of PFN capacitors and geometric size of the magnet components) the number of cells has been chosen to be 24 and 27 for the PFN's and the magnet respectively.

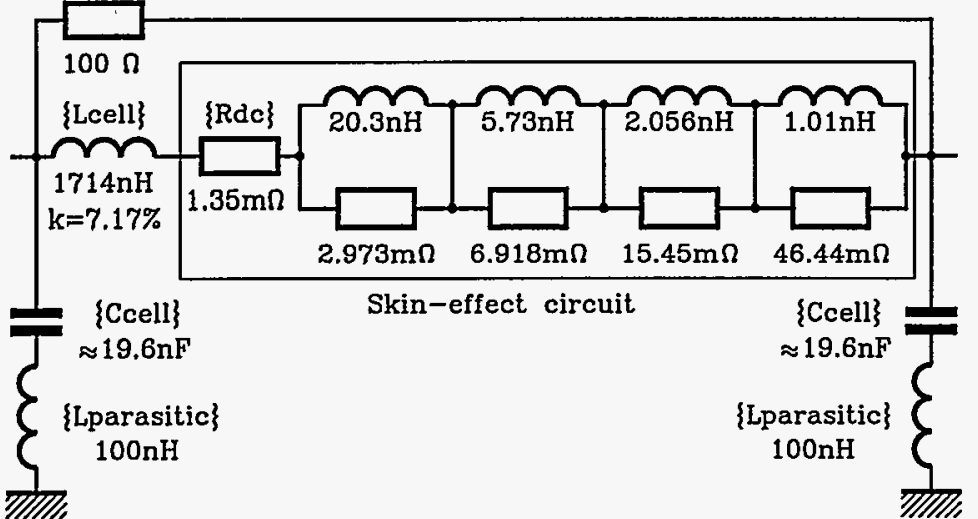

Fig. 2: Equivalent circuit for a central PFN cell

Fig. 2 shows the equivalent circuit simulated for each central cell of the PFN's: Lcell represents the series inductance of the cell, Ccell the shunt capacitors, and Lparasitic the associated parasitic inductance. The nominal value of shunt capacitor was defined as $\mathrm{Ccell}=\mathrm{Lcell} \cdot(1+2 \mathrm{k}) /(2 \mathrm{Z})^{2}$, where $k$ is the coupling coefficient between adjacent cells. A $100 \Omega$ damping resistor which is effective at helping to reduce the ripple was connected in parallel with each central PFN cell. In order to obtain a realistic prediction it was necessary to simulate skin-effect for each cell of the PFN; proximity effect has not yet been represented. Skin-effect from almost $\mathrm{DC}$ up to a frequency of $10 \mathrm{MHz}$ has been simulated using a series resistance and four inductor-resistor networks (Fig. 2). $\{\mathrm{Rdc}\}$ represents the DC resistance of the PFN cell, assuming a copper tube of $\varnothing 8 \mathrm{~mm}$, and wall thickness of $1 \mathrm{~mm}$, for the coil. Each of the four inductor-resistor networks predominantly affects a different part of the frequency range of interest. Analytical equations were utilised to determine initial guesses for the resistor-inductor networks. The values of these elements were then refined using the PSpice Optimizer ${ }^{[4]}$. The optimised values resulted in an excellent fit to the calculated skin-effect resistance.

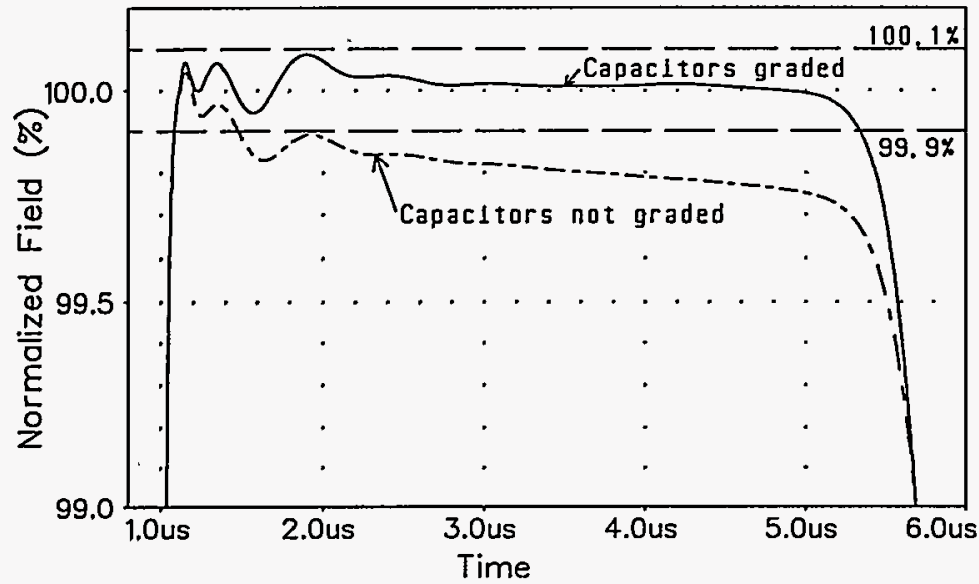

Fig. 3: Effect of grading capacitor in PFN to compensate for conduction losses
Conduction losses along the coil result in droop of the top of the magnetic field pulse in the kicker magnet (see Fig. 3). These losses can be compensated for by grading the values of the capacitors such that they increase in value from the MS end to the DS end of the PFN's. Linear grading throughout the PFN's is suitable: the optimum linear grading factor is $0.075 \%$ per cell, i.e. for a 24 cell PFN the value of the shunt capacitor at the DS end is $1.74 \%$ greater than the nominal value of shunt capacitor at the MS end.

It is planned to use the natural production spread of capacitor values to select the precise graded value required. To check this possibility a batch of about 140
ysica ${ }^{[6]}$ macro has been written which selects capacitors with a nominal value of $20 \mathrm{nF} \pm 5 \%$ has been measured. A Physica ${ }^{[6]}$ macro has been written which selects
pairs, from the batch of capacitors, whose values closely sum to the required graded values. Each of the two capacitors of a pair was simulated as being in corresponding positions in the two PFN's: however the capacitors were also arranged such that the running average values in both PFN's are approximately equal. In order to achieve a characteristic impedance of $10 \Omega$, a cell self-inductance and nominal shunt capacitance of $1714 \mathrm{nH}$ and $19.6 \mathrm{nF}$, respectively, were chosen. The available batch would allow the use of more than $80 \%$ of the capacitors for building PFN's with the required characteristics: this gives us confidence that the PFN's can be built from a batch of commercially available capacitors.

To control the path of the return current and contain the magnetic field a coaxial copper screen will surround the coil. Each cell of the PFN is mutually coupled to adjacent cells. The value of coupling coefficient $k$ between cells depends on the ratio between screen and coil diameter: e.g. $k=7.17 \%$ and $9.5 \%$ for ratios of 3 and 100 , respectively. 
It is desirable to minimize this ratio, and hence the volume of the tank. PSpice simulations showed that the $\pm 0.1 \%$ specification for field quality can be achieved with $k=7.17 \%$. Thus the ratio of 3 has been retained for the construction.

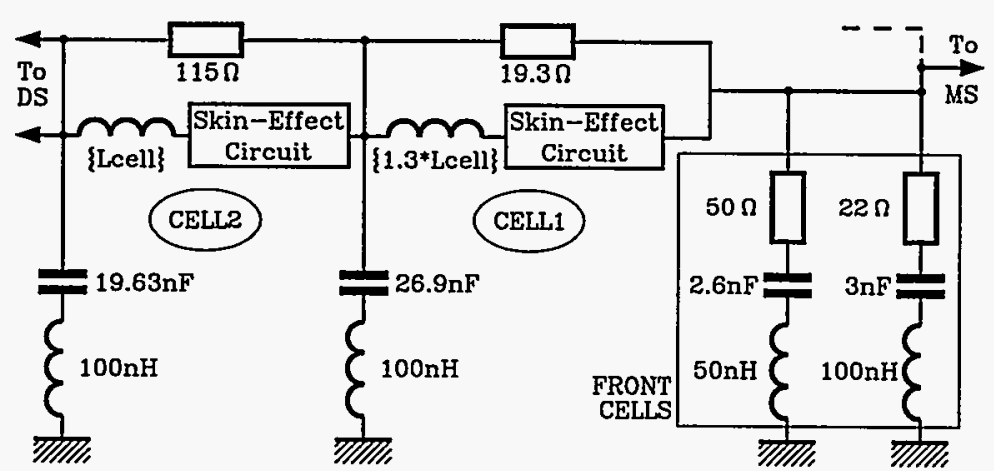

Fig. 4: Main switch end of the PFN
- End cells

The end cells of the PFN's need component values different from those of the central cell (Fig. 4). Cell 1 has a $30 \%$ increased self-inductance, approximately a $37 \%$ increased cell capacitance, and a parallel resistor of $19.3 \Omega$. Similarly Cell 2 has a parallel resistor of $115 \Omega$. In addition, two R-C networks (front cells) are required: their time constants were chosen for optimum pulse response.

The post-pulse period is only critical for the last of the 12 injection pulses which has a reduced flat top duration of $4.25 \mu \mathrm{s}$.

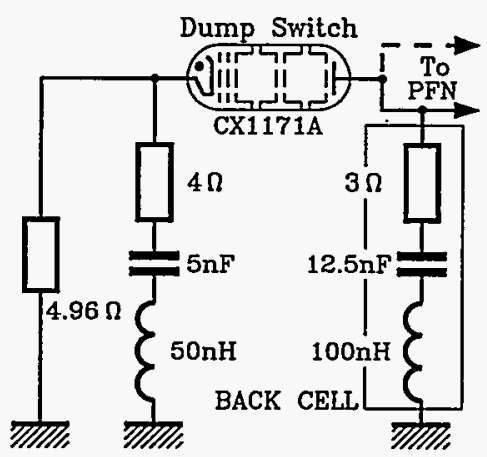

Fig. 5: Dump switch end of the PFN The DS is only required for the shorter pulse. The post-pulse period is affected by both the pulse launched by the DS and by impedance mismatches at the DS end of the PFN, which causes a proportion of the wavefront launched by the MS to be reflected back towards the MS. In order to ensure that the impedance at the DS end of the PFN is matched over the frequency range of interest, PSpice AC analyses were carried out which show that the DS end needs a series connected R-C network (back cell). A second R-C circuit is required in parallel with the DS termination resistor. Subsequently PSpice transient analyses were performed, using the circuit components determined from the $\mathrm{AC}$ analyses as a starting point. The optimised values for the back-cell are indicated in Fig. 5. As a result of the need to grade the PFN capacitors the characteristic impedance of the two parallel PFN's, as seen by the DS thyratron, is approximately $4.96 \Omega$ : hence the DS termination resistor has a value of $4.96 \Omega$. The predicted fall time is $2.91 \mu$ s $(99.9 \%$ to $0.1 \%)$.

In order to assess the effect of displacement current from the CX 1171 thyratron during turn-on, a mathematical model ${ }^{[5]}$ of the three-gaps and two drift spaces of the CX 1171 was utilised. As a result of both the relatively low characteristic impedance of the kicker system and the relatively long fill time of the kicker magnet $(\sim 525 \mathrm{~ns})$, it was shown that the thyratron displacement current does not significantly affect the $0.1 \%$ to $99.9 \%$ field rise-time.

\section{Kicker Magnet}

The above reported simulations for optimising the PFN modelled an ideal transmission line kicker magnet. In reality the finite number of cells and the parasitic inductances of the capacitors reduce the cut-off frequency of the magnet. Hence a realistic model of each cell of the kicker magnet (Fig. 6) has been used, together with the optimised PFN, to assess the field quality. The nominal self-inductance and capacitance of each cell of the kicker magnet were modelled as $101.2 \mathrm{nH}$ and $4.04 \mathrm{nF}$, respectively. The ferrite was modelled as having a quality-factor $Q=5^{[7]}$.

A ceramic tube with ten $5 \mu \mathrm{m}$ thick copper stripes sputtered onto the inner wall will be placed in the aperture of the magnet. The stripes provide a conducting path for the image current of the beam, and, in combination with the pipe dielectric, screen the ferrite against the wake fields ${ }^{[8]}$. The stripes are connected via decoupling capacitors to the standard vacuum chambers of the machine.

In the mathematical model each stripe was broken down into 27 equal parts, one part for each cell of the magnet. The inductance of each part was mutually coupled to the selfinductance of the corresponding cell of the kicker magnet. The coupling coefficient was simulated as reducing linearly from $27.2 \%$, for the stripe adjacent to the high-voltage busbar, to $4.4 \%$ for the stripe adjacent to the ground busbar. Inter-stripe capacitance is also simulated.

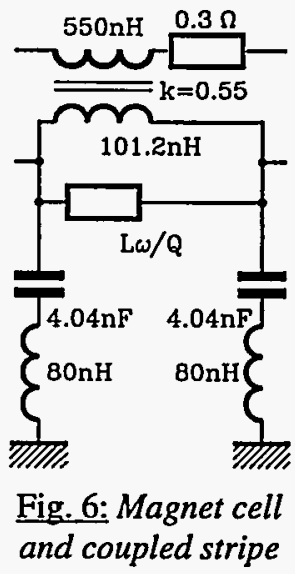


The 10 stripe model of the kicker magnet is relatively complex, and needs significant CPU time to analyse its performance. Hence analytical equations were derived which show that, provided all stripes have the same selfinductance, series resistance and decoupling capacitance, and neglecting inter-stripe capacitance, the 10 stripes may be replaced with a single stripe as shown in Fig. 6. The coupling coefficient between the main inductance of the cell and the stripe is equal to the root of the sum of the squares of the 10 coupling coefficients, i.e. 0.55 . The simplified single-stripe representation required approximately $5 \%$ of the CPU time required for the full circuit, and the predicted field pulses were in excellent agreement.

Using the simplified single-stripe representation of the metallization, $\mathrm{R}-\mathrm{C}$ networks at the input and output of the kicker magnet have been optimised. This process was carried out for different values of decoupling capacitances between each end of the stripe and ground. The results indicate that the R-C network can effectively be re-optimised for any decoupling capacitor below $200 \mathrm{pF}$.

Fig. 7 shows the predicted field pulse with each of 10 stripes capacitively coupled to ground with $60 \mathrm{pF}$ at each end of the tube, and with optimised R-C networks at the input and output of the magnet. The predicted field rise-time and flat-top ripple are both within the design specifications. The post-pulse prediction is very similar to that of an ideal transmission line magnet.

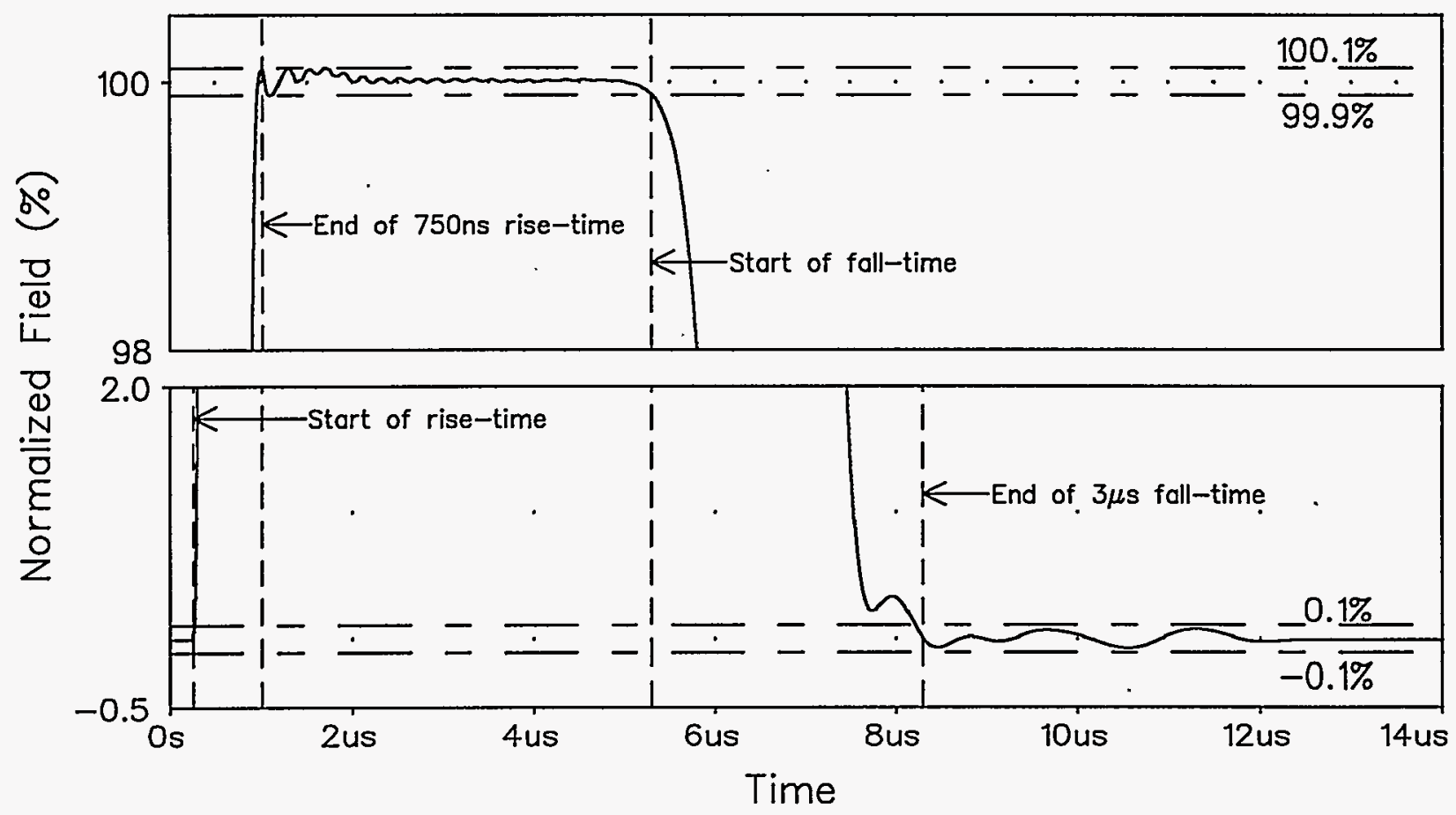

Fig. 7: Predicted magnetic field kick for the short pulse, with the optimised equivalent circuit

\section{Mechanical design}

The two PFN's are housed in the same metallic tank and insulated with silicone fluid. The capacitors are mounted vertically and in individual coaxial housings. The two straight and continuous copper coils are wound around a threaded glass reinforced epoxy cylinder and locked by outer tubes to ensure a precise and constant geometry along the coil. A series of holes through the cylinder and the outer tubes allows connections to be made to the capacitors which are mounted below and in line with the coil. Each coil is shielded by a copper screen connected to the coaxial housing of the capacitors. The coils are connected in parallel, and then to the switches via a common coaxial "plug-in" feed-through.

Main and dump thyratron switches will be installed in independent tanks with isolating transformers for heaters, reservoirs and grid biasing. These switches will be mounted directly onto the PFN, which is a compact and cost efficient solution already in use at CERN for the SPS injection system.

Both matching resistors will be assembled from ceramic-carbon discs mounted in a low inductance coaxial housing and insulated with silicone fluid. This type of resistor is extensively used at CERN, but the adjustment facility and the resistance stability must be improved for this very precise application. It is intended to implement a 
temperature regulation on the cooling circuit to control the nominal resistance value. The temperature coefficient of the resistor discs is $0.1 \% /{ }^{\circ} \mathrm{C}$.

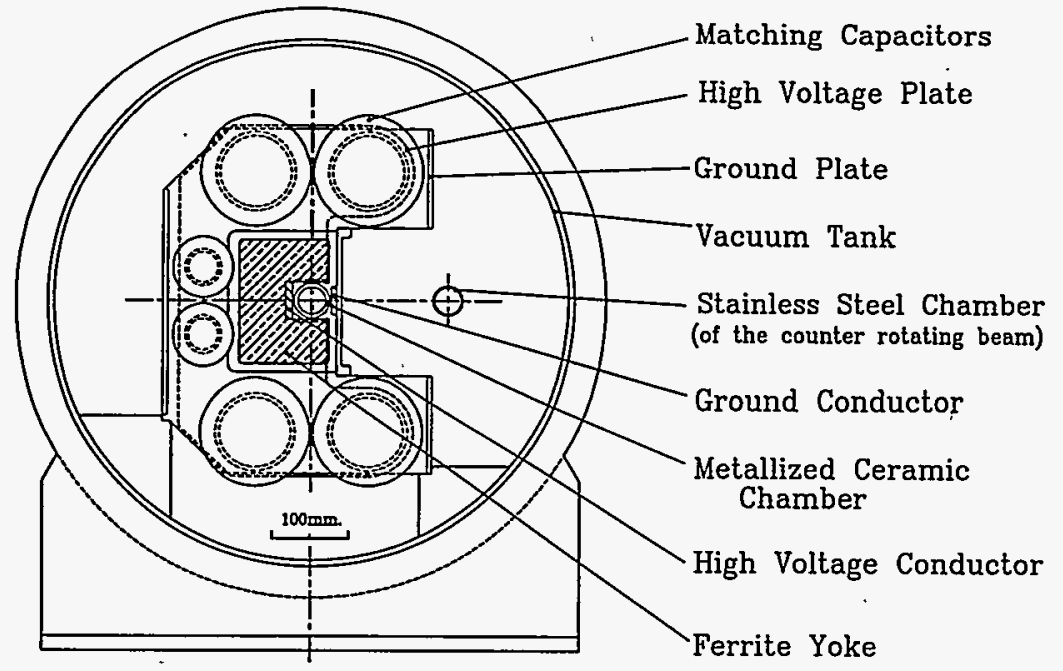

Fig. 8: Schematic cross section of the magnet
Fig. 8 shows a schematic cross section of a magnet cell with $\mathrm{C}$-shaped ferrite yoke and 6 ceramic matching capacitors mounted between high voltage and ground plates. The relatively long field rise time allows one to build a magnet of $2.2 \mathrm{~m}$ length. The capacitance of $4.04 \mathrm{nF}$ per cell will be achieved by means of high voltage/high frequency grade 1 ceramic plate capacitors. Outgassing tests have shown that these capacitors can be used under high vacuum provided that clamped connections are used instead of the usual brazed ones. The magnet will be housed in a cylindrical vacuum tank of about $600 \mathrm{~mm}$ diameter.

\section{Conclusion and further developments}

The simulation results have shown that it is theoretically possible to achieve the required magnetic field pulse with a flat top ripple and reflections of less than $\pm 0.1 \%$. Nevertheless, some parasitic effects have not yet been incorporated in the equivalent circuit, e.g. capacitances between adjacent turns of the PFN coil and a more accurate model of the termination resistors. These elements will also be simulated. It should be possible to compensate their effect by re-optimising the front cells of the PFN's and the R-C networks of the magnet. Furthermore, the components of the equivalent circuit will be submitted to systematic variations in order to study effects such as ambient temperature changes and ageing of capacitors. The predictions will show the real limitations of the system, which we expect to be close to the required performances of $\pm 0.5 \%$.

Mechanical design of a full scale prototype has started and assembly of PFN's and switches is foreseen for early next year.

\section{Acknowledgement}

We would like to thank our colleague Pearce for his help in the mechanical development of the system.

\section{References}

[I] E. Frick, H. Kuhn, M. Mayer, V. Rödel. G.H. Schröder, E.B. Vossenberg, "Fast Pulsed Magnet Systems for Proton and Antiproton Injection into the CERN $400 \mathrm{GeV}$ Proton Synchrotron", 15th Power Modulator Symposium, Baltimore, June 14-16, 1982.

[2] Communication with E. Decailloz, PFN design Department, LCC-Thomson, France, 1993.

[3] Hydrogen Thyratrons (Product Data). English Electric Valve Co., Chelmsford, Essex, UK.

[4] MicroSim Corporation, 20 Fairbanks, Irvine, California 92718. USA Tel (714) 7703022.

[5] M.J. Barnes, G.D. Wait, "A Mathematical Model of a Three-Gap Thyratron Simulating Turn-On", Proc. of the 9th IEEE International Pulsed Power Conference, June 21-23, 1993. pp 293-296.

[6] J.L. Chuma, "Physica Reference Manual", TRI-CD-93- 01, November 1994.

[7] G.D. Wait, M.J. Barnes, H.J. Tran, "Magnetic Field in a Prototype Kicker Magnet for the KAON Factory", IEEE Trans. on Magnetics, July 1994, Vol. 30, No. 4, pp 2118-2121.

[8] A. Piwinski, "Penetration of the Field of a Bunched Beam through a Ceramic Vacuum Chamber with Metallic Coating", IEEE Transaction on Nuclear Sciences. NS-3, 1977. 